Relations industrielles

Industrial Relations

\title{
The Winnipeg General Strike, par D.G. Masters, Toronto, University of Toronto Press, 1973, 159 pp.
}

\section{Gérard Dion}

Volume 29, numéro 1, 1974

URI : https://id.erudit.org/iderudit/028494ar

DOI : https://doi.org/10.7202/028494ar

Aller au sommaire du numéro

Éditeur(s)

Département des relations industrielles de l'Université Laval

ISSN

0034-379X (imprimé)

1703-8138 (numérique)

Découvrir la revue

Citer ce compte rendu

Dion, G. (1974). Compte rendu de [The Winnipeg General Strike, par D.G. Masters, Toronto, University of Toronto Press, 1973, 159 pp.] Relations industrielles / Industrial Relations, 29(1), 231-231.

https://doi.org/10.7202/028494ar

Tous droits réservés (C) Département des relations industrielles de l'Université Laval, 1974
Ce document est protégé par la loi sur le droit d'auteur. L’utilisation des services d'Érudit (y compris la reproduction) est assujettie à sa politique d'utilisation que vous pouvez consulter en ligne.

https://apropos.erudit.org/fr/usagers/politique-dutilisation/ 
cherchée, Bercuson introduit à la fois «l'étude des principes sous-tendant la reconstruction du monde des relations du travail » et un Mackenzie King, réformiste, qui tente d'indiquer la voie d'un changement d'attitude dans les relations industrielles et de suggérer des moyens par lesquels un nouvel esprit pourrait pénétrer l'industrie.

En plus d'un large exposé pour l'établissement d'un nouveau programme de démocratie dans le monde industriel, l'ouvrage passe en revue plusieurs crises d'importance dans les relations patronales-ouvrières de l'Amérique du Nord et révèle l'arrière-plan de multiples pièces de la législation canadienne en matière de sécurité sociale.

Lorsqu'on fait abstraction de l'attitude paternaliste de King et des rumeurs à l'effet qu'il soit l'un des auteurs du plan Mohawk, véritables «tables de la loi » et programme détaillé de la répression à l'égard des travailleurs en grève, Mackenzie King apparaît comme un des penseurs, dignes d'intérêt, en matière de relations du travail. Qu'il nous suffise de souligner que pour lui, et c'est là un des fondements de sa thèse, le monde industriel comporte non pas deux (2) ou trois (3) mais quatre (4) parties: le travail, le capital, le management et la communauté. Croyant que la démocratie et la paix industrielles puissent reposer sur la bonne foi, le raisonnement et la compréhension mutuelle, il explique qu'une bonne part de la tension, le malaise et l'injustice sociale sont crées simplement par le fait que travail et capital ne réussissent pas ou refusent de reconnaître la légitimité des intérêts des deux autres parties. Partant de là, il énonce des principes que le temps et l'expérience sauraient mettre à l'épreuve notamment que les conflits trouveraient solution par le biais d'enquêtes conjointes (où seraient présentes les quatre (4) parties) utilisant des méthodes scientifiques tout comme les crises internationales pourraient être résolues à la Société des Nations (!!!).

Ce sont là des pensées que d'aucuns pourront qualifier de pédantes, moralistes ou même farfelues, mais qu'il vaut sûrement la peine de lire dans un ouvrage qui pour certains constitue un classique.
On doit cependant prévenir le lecteur que les longueurs, les raisonnements tortueux et le style lourd de King rendent l'ouvrage difficile à lire, mais cela ne devrait pas décourager celui qui s'intéresse à l'évolution de la pensée des systèmes industriels car qu'on le veuille ou non, William Lyon Mackenzie King a écrit quelques pages de notre histoire.

Jean-Louis LANGEVIN

Université Laval

The Winnipeg General Strike, par D.G. Masters, Toronto, University of Toronto Press, 1973, 159 pp.

La grève générale de Winnipeg a été un événement important dans l'histoire du syndicalisme au Canada. En raison de son caractère syndical et politique, elle a donné lieu à de nombreuses études et à diverses interprétations.

L'ouvrage de D.C. Masters publié en 1950 demeure le classique sur le sujet. Epuisé depuis plusieurs années, il faut se réjouir de constater que les éditeurs aient décidé d'en faire une nouvelle édition brochée à prix populaire.

On s'est contenté de reprendre exactement le même texte en ajoutant seulement une brève préface de l'auteur dans laquelle celui-ci apporte quelques précisions sur des points de détail controversés.

Gérard DION

\section{Université Laval}

Labour Economics: Wages, Employment and Trade Unionism, by Allan N. Cartter and F. Ray Marshall, Homewood, Illinois, Richard D. Irwin, Inc., (Revised edition), 1972, 594 pp.

This book is a revised edition; the first edition appeared in 1967. It is aimed at the introductory labour economics and industrial relations courses. The book is divided into three major sections: comparative labour movements (about 147 pages); wages, employment and collective bargaining 